\title{
Player Behavioral Tracking and Personalized Feedback in On- line Gambling: Implications for Prevention and Treatment of Problem Gambling
}

Keywords: Online gambling; Problem gambling; Responsible gambling; Pop-up messages; Limit-setting; Behavioral tracking; Personalized feedback; Prevention; Treatment

\begin{abstract}
In recent years, participation in online gambling has been growing at a significant rate, as have concerns over its connection to problem gambling. The relationship between online access and problem gambling is complex. On one hand the nature of online gambling appears to invite potential for problematic gambling; on the other hand it is also well-suited to the incorporation of sophisticated RG tools that can help prevent excessive gambling. This article provides a brief overview of research on the efficacy of several increasingly available RG tools - limit-setting, in game "pop-up" messages and player behavioral tracking and personalized feedback - and the potential of these tools, used in concert, to increase gambler's self-awareness and self-monitoring to reduce risk across a broad spectrum of gambling involvement. In addition to weighing the growing evidence for the preventative utility of such RG strategies, we also consider the burgeoning interest in their therapeutic/clinical potential, both as part of self-guided or therapist-assisted online interventions and as adjunct to conventional in-person clinical services. We conclude that, limitations notwithstanding, the evidence for online RG tools that incorporate player behavioral tracking and personalized feedback is quite encouraging when it comes to reducing problem gambling risk and that, although less far along, research on the treatment potential of interventions incorporating such technology is also promising and merits further study.
\end{abstract}

\section{Introduction}

A shift towards Internet gambling in recent decades has coincided with growing concerns regarding problematic gambling behavior [1]. In fact, a survey performed by the British Gambling Commission found that almost nine percent of surveyed adults had participated in some form of online gambling [1]. Similarly, a prevalence study conducted in Australia, found over eight percent of survey respondents had gambled online [2]. In a study that analyzed British youth online gambling prevalence, Griffiths and Wood found that eight percent of children aged twelve to fifteen had "played a national lottery game over the internet" $[1,3]$. Online gambling is not yet as popular in Canada, where the national online prevalence rate was slightly higher than two percent in 2007, reaching as high as four percent in some provinces $[4,5]$. The global market for Internet gambling is growing despite various government restrictions in countries such as the United States [6]. Like Canada, the United States had an online gambling prevalence rate slightly higher than two percent in 2011, and that number is expected to grow over time [7]. Furthermore, a study conducted by Welte and colleagues found
Journal of

Addiction \& Prevention
Jason D. Edgerton*, Jeff Biegun and Lance W. Roberts

Department of Sociology, University of Manitoba, Manitoba, Canada

\section{*Address for Correspondence}

Jason D. Edgerton, Department of Sociology, University of Manitoba, 323 Isbister Building, Winnipeg, Manitoba, Canada, Tel: 204-474-8192; Fax: 204-2611216; E-mail: J.Edgerton@umanitoba.ca

Submission: 22 September, 2016

Accepted: 01 November, 2016

Published: 10 November, 2016

Copyright: ๑ 2016 Edgerton JD, et al. This is an open access article distributed under the Creative Commons Attribution License, which permits unrestricted use, distribution, and reproduction in any medium, provided the original work is properly cited.

that online gambling was the only gambling form that saw an increase in its prevalence rate, where traditional forms such as lotteries saw a decrease [7]. In short, participation in online gambling is growing at a significant rate, as are concerns over its connection to problem gambling [8].

Given the risks associated with excessive gambling and the global proliferation in the availability of legalized gambling [8], there has, in recent years, been growing recognition of the importance of promoting responsible gambling $[9,10]$. Responsible gambling (RG) strategies aim to encourage players to gamble within their means, reducing the risk of negative personal and social consequences [11]. Because the majority of gamblers are low risk recreational gamblers, RG (or harm minimization) tools can help them manage risk and maintain control of their gambling activity within affordable time and monetary limits, and can also provide a harm-reduction alternative to abstinence for players who have experienced or may be at risk for gambling problems [12-14]. The increase in legalized gambling opportunities has been accompanied by an increase in the availability of RG tools for gamblers. Prevailing RG strategies include provision of informational and warning messages to players (including signs and in-game "pop-ups") and pre-commitment to time and monetary limits. Player account data from online gambling operators also holds promise of complementing and enhancing these tools with behavioral tracking and personalized feedback $[12,15,16]$.

A comprehensive review of the causes and mechanisms underlying problem gambling and the relationship between online gambling and problem gambling is beyond the scope of the present article [17-21]. There is substantial evidence pointing to an association between online gambling and problem gambling, but the nature of this relationship is complex and requires further study before possible causal links can be clarified. In brief, there are few personal or behavioral characteristics that reliably distinguish Internet from non-Internet problem gamblers, as there is much heterogeneity within and overlap between the populations. On one hand, online gambling would appear to invite increased risk of gambling problems given its immersive nature, wide-spread availability (including 
anytime anywhere access via mobile and wireless devices), ease and convenience of play (including tutorials and free-play modes, electronic payment), and the solitary and anonymous nature of online play and the ability to play, undetected, under the influence of substances; on the other hand, there is evidence that when other relevant variables such as frequency of gambling activity and number of different types of gambling activity engaged in are controlled for, Internet gambling is associated with no additional risk [20,22-25] Problem gamblers are more likely to gamble online because they are more likely than non-problem gamblers to engage in a variety of gamble activities, both online and offline, with greater intensity. Research suggests the clinical characteristics - including mental health comorbidities (e.g. mood and substance use disorders), impulsivity, and irrational and erroneous cognitions - of online problem gamblers are similar to land-based problem gamblers [21]. Given the capacity to collect and track player behavioral data and to deliver salient ingame messages, online gambling is well-suited to incorporating various individually tailored RG tools and may actually be associated with decreased risk for some gamblers [10,26-28].

In this article we provide a brief overview of research on the efficacy of several increasingly available RG tools - limit-setting setting, in game "pop-up" messages and player behavioral tracking and personalized feedback - and the potential of these tools, used in concert, to increase gambler's self-awareness and self-monitoring to reduce risk across a broad spectrum of gambling involvement, including gamblers looking to sustain non-problem levels of gambling and more at-risk gamblers who are looking for alternatives to abstinence $[12,14]$. In addition to weighing the growing evidence for the preventative utility of such RG strategies, we also consider the burgeoning interest in their therapeutic/clinical potential, both as part of self-guided or therapist-assisted online interventions and as adjunct to conventional in-person clinical services.

\section{In-Game Pop-up Messages}

A significant amount of attention has been paid to the use of popup messages in online gaming sites to promote RG and minimize gambling-related harm. It is common for problem gamblers to dissociate while gambling, losing track of time and place and the immersive, solitary nature of online gambling would seem to further invite such disassociation [29,30]. Monaghan discussed how popup messages can serve to briefly interrupt individuals' dissociative immersion in the flow of game play, drawing their attention to harm minimization messages and increasing self-awareness and self-monitoring of their gambling behavior [24]. In-game pop-ups do not demand a user actively seek out RG information, and do not require users to divide their attention across competing sources of information (playing the game while reading information on harm minimization strategies on a separate website or a poster, for example).

In a laboratory study, using a commercial electronic gaming machine, Monaghan and Blaszczynski found that $83 \%$ of participants exposed to in-game "dynamic" pop-ups were able to accurately recall the content of the messages, compared to a recall rate of only $15.6 \%$ for "static" warning signs [31]. Monaghan and Blaszczynski found that, in both laboratory and non-laboratory settings, pop-up messages were significantly more likely to be recalled than static messages, and that pop-ups had significantly greater self-reported influence on insession gambling behavior (e.g. taking a break in play, changing their betting pattern) [31]. These results are similar to previous studies that found that pop-ups are associated with decreases in the duration of gambling sessions and consequent monetary expenditures [32,33]. For example, Cloutier et al. had previously explored the impact of pauses and pop-ups as a risk management tool for gamblers [34]. Using an undergraduate sample of participants who engaged with a "simulated bar", the authors exposed participants to game pauses and/ or pop-up messages, with a control group being exposed to neither [34]. They found that both messages and pop-ups were effective at reducing erroneous thoughts and increasing cessation of gambling, but that pop-ups were more effective.

Additionally, Monaghan and Blaszczynski found that the content of pop-up messages is important [31]. Pop-ups that encourage self-appraisal are more effective than informational pop-ups. Informational pop-up messages include those that provide the user with information related to the probability of winning the game they are playing ("Your chances of winning the maximum prize are generally no better than one in a million" or "All outcomes are randomly determined by chance"). Self-appraisal messages encourage participants to actively reflect on their behavior in the on-going gambling session and whether they might need a break ("Do you know how long you have been playing? Do you think you need to think about a break?" or "Have you spent more than you intended? Do you need to think about a break?") [31]. While immediate recall by participants showed no difference between pop-up message content types, after a two-week period recall was higher for self-appraisal pop-up message content than it was for informational pop-ups. Furthermore, the authors report that participants exposed to self-appraisal messages were more aware of their time and monetary investment, and were better able to estimate their own chances of winning and losing.

Taken together, the research above suggests that traditional forms of RG messaging, such as warning signs and stickers, or having information located separately from the gaming source itself, are less effective at conveying information that is retained by users. RG information delivered via "dynamic" in-game pop-ups breaks up the disassociation process that players often experience while gambling and encourages mindfulness and self-monitoring (via self-appraisal prompts) and is more likely to be retained and to influence gambling behavior than are static warning signs and stickers.

\section{Pre-setting Limits}

Pre-commitment to voluntary time and money limits is an increasingly widespread RG practice being adopted by online gaming operators [35]. Pre-setting limits is seen as another strategy to help disrupt the dissociation that gamblers often experience in which they lose track of time and money spent in prolonged gambling sessions. Problem gamblers are particularly prone to spending more than they intend [36]. Although empirical evidence on the effectiveness of various pre-commitment programs is still somewhat mixed there is growing consensus that such features are highly desirable and should be given prominence in RG policy considerations by regulators and operators $[1,9,10,16]$.

Limits can be voluntary or mandatory, operator defined or player defined [16]. Types of spending limits include deposit limits (amount a 
player can deposit into their account at one time), play limits (amount a player can play with at any one time), loss limits (amount a player is allowed to lose in any one session), or bet limits (amount that a player can bet in any one game or session) [35]. Player defined limits seem to be the more effective than operator imposed limits in preventing players from spending too much time or money, particularly among the most intense players who are most at risk $[10,12,37]$.

A number of recent studies have looked at augmenting precommitment strategies with pop-ups. Although generally used less than monetary limits, time limits can be helpful for some players to reduce the duration of gambling and the probability of excessive gambling [38]. Kim and colleagues explored a form of enhanced popup that asks users to consider pre-setting a time limit at the beginning of their gaming session [39]. Those participants were more likely to set a limit, and to spend less time gambling than participants who did not receive the instructions. The authors suggest that the very act of considering and pre-setting a time limit may have caused the players to be more conscious of monitoring their playing time which may result in shorter play duration and less risky play.

Stewart and Wohl examined the effect of pre-determined spending limits reinforced by reminder pop-ups [40]. They found that participants who received a pop-up reminder of their selected spending limit where significantly more likely $(89.66 \%)$ to keep to that limit than were players who did not receive a pop-up prompt (43.33\%). Wohl and colleagues looked at the effectiveness of combining educational animations (either a 'neutral' video or one explaining how EGMs work) with spending-limit reminder pop-ups [41]. They found that both the pop-ups reminding players of their pre-selected spending limit and educational animations were effective at increasing the likelihood that players would observe their chosen spending limit. Interestingly there was no additive effect, combining the educational animation with the pop-ups did not increase player compliance, and so the authors recommend pop-ups as preferable over the lengthier educational animation.

Noting that most previous studies of pop-ups relied on selfreport in laboratory or simulated settings, Auer et al. assessed the effectiveness of pop-ups with real world data [42]. They wanted to test whether pop-up messages could successfully terminate prolonged gaming sessions on online slot machines. Using anonymized player data provided by an online gambling site operator, they tracked data from approximately two-hundred thousand gamblers across two random samples of approximately four-hundred thousand gambling sessions. One RG feature of the gambling site that the data came from was that after one thousand spins (a playing time of approximately 60 minutes) on the online slot machine, users are presented with a popup informing them "You have now played 1,000 slot games. Do you want to continue? (Yes/No)". The pop-up remains on screen until the player answers, if they answer "Yes" the pop-up disappears and they can continue, if they answer "No" the gaming window closes. While Auer et al. suggest caution in interpreting their results, as they are unable to verify who saw the pop-up and who did not, there were a significant number of game terminations around the one-thousandth spin - the point where users would have been prompted with the popup [42]. After the one-thousandth spin, games ended at a rate nine times higher than before the appearance of the pop-up-suggesting the pop-up was a compelling cue for some gamblers.

As a follow-up study using data from the same site, Auer and Griffiths examined whether additional "enhanced" pop-up content was more effective than simple (non-enhanced) pop-ups [43]. The "simple" pop-up contained the same message as the earlier study, informing users they had played for one-thousand spins and asking them if they would like to continue or not. The "enhanced" pop-up provided information not only about the duration of play ("We would like to inform you, that you have just played 1,000 slot games"), but also about the chances of winning ("The chance of winning does not increase with the duration of the session"), as well as normative ("Only a few people play more than 1,000 slot games") and self-appraisal information ("Taking a break often helps, and you can choose the duration of the break"). Enhanced pop-ups doubled the number of gamblers who quit their gambling sessions, but the effect was rather modest with $1.4 \%$ of highly involved gamblers (those who played 1000 consecutive slot games) immediately ceasing their gambling session after receiving the enhanced pop-up. It should be noted that the feedback messages given to gamblers were still relatively generic and other than noting they had played 1000 consecutive games did not involve any customized indicators of the players' actual gambling activity in that session or across sessions. The research covered in the next section attempts to address the possibility that more personalized feedback may have greater effect in changing gambler behavior.

\section{Player Behavioral Tracking and Personalized Feedback}

\section{Prevention}

Recently, attention has increasingly turned to whether RG tools, such as pre-commitment and pop-ups, combined with player behavioral tracking data to provide more personalized user feedback, could help players to actively monitor and better regulate their own gambling behavior within affordable, non-harmful, limits.

There is evidence to suggest that certain behavioral markers (e.g. betting more frequently, high variability in wager sizes, chasing losses, exceeding pre-set limits, etc.) are predictive of future gambling problems, and that problematic patterns of gambling are often evident before the emergence of gambling-related harm [44-49]. Online gambling site operators have the capacity to collect behavioral information - such as types of games played, frequency and duration of gambling sessions, amounts deposited, bet and wagered, use of RG tools, etc. - that can track player gambling patterns within and across sessions. Such behavioral tracking can offer means of early detection of at-risk gambling and the possibility of providing access to minimally invasive harm minimization tools to pre-empt problematic play and/or loss of control [26]. Such tools, informed by sophisticated algorithms can detect potentially problematic behavior proactively triggering harm minimization features customized to the needs and preferences of the player [15]. Given important variations in subgroups of gamblers in terms of activities, motivations and issues, such individualization of harm reduction features can help optimize their impact and effectiveness [50].

It appears that most players welcome harm minimization tools as long as they are able to exercise consumer choice via player-initiated RG features such as pre-setting limits themselves [51]. In addition to player defined limits, other recommended RG features for online 
gambling include provision of detailed behavioral information (e.g length and frequency of players' past sessions) and real time visible displays or pop-ups that indicate time spent playing and amounts won and lost, as well as warnings of risky changes in behavior [10] Such continuous feedback respects consumer choice and empowers gamblers to exercise informed self-regulation and risk-management. This emphasis on providing players with the information necessary to make informed decisions about their own gambling participation is increasingly at the forefront of RG initiatives in that emphasize a "duty of care" on the part of gambling operators [12,52-54].

Wohl and colleagues suggest that the effectiveness of popup messages and related harm-reduction tools can be improved by applying Human Computer Interaction (HCI) and Persuasive Systems Design (PSD) principles [55]. A core HCI principle is that feedback from end users must be incorporated in every stage of design; other principles include aesthetically appealing design, statusupdate tools that give ongoing feedback to users (e.g. how much money is left in gambling account), tools that promote functionality and user control (e.g. self-limit setting tools), clearly articulated information and instructions (e.g. how to pre-set a monetary limit). Relevant PSD principles include tunnelling (i.e. lead users through sequence of steps to reach their objectives), tailoring (i.e. provide design or information targeted to help specific users reach their objectives), suggestion (appropriately timed prompts to help facilitate their objectives), and self-monitoring (i.e. providing users with tools to help them actively manage their own progress toward their objectives). Wohl et al. incorporated these HCI and PSD principles into the design of an aesthetically appealing monetary limit-setting tool to test whether it would be more effective in compelling gamblers to adhere to their pre-set limits [55]. Notable features of the monetary limit tool included: easily accessible player stats and a traffic light metaphor warning system that changed from green to yellow as the player neared their limit (within 10\%) and then turned red when the limit was reached; pop-up messages that alerted the player when they had only $10 \%$ of their limit remaining, and again when they actually reached their limit; a 5 second delay before play could continue after the $10 \%$ pop-up message, and a 1 minute delay after the limit reached message. Results showed that significantly more (92\%) of participants exposed to the HCI and PSD inspired monetary limit tool complied with their pre-set money limits compared to a control group (62.2\%) exposed to a traditional monetary control tool.

While Wohl et al. used undergraduates in a laboratory setting, Wood and Wohl were able to use data from a actual gambling website to assess whether receiving feedback about a potentially problematic change in their play would influence the amounts deposited and wagered by players $[55,56]$. The Swedish state-owned gambling operator Svenska Spel offers its customers the option of using the RG tool Playscan. Players who sign up receive behavioral feedback in the form of a traffic light signal, with green signifying no problem, yellow signifying some risky play and red signifying problematic play-the risk level is assigned by the behavioral tracking software (via a proprietary algorithm). The authors compared the group that used behavioral feedback with a matched group that did not on amounts deposited and wagered at 3 points in time. They found that non-risk and at-risk players who used the tool significantly decreased their spending compared to players who didn't receive feedback. No such differences were evident for high risk gamblers. The authors suggest that behavioral feedback might be more useful as a preventative aid for helping at-risk players moderate their gambling behavior before it gets out of control, rather than as a treatment intervention for high risk gamblers. An important caveat to this conclusion is that it is not known how the Playscan risk level classifications compare to more standardized measures of problem gambling such as the Canadian Problem Gambling Index or DSM criteria.

\section{Personalized feedback interventions}

Personalized feedback interventions (PFI), which are rooted in social learning theory and the social norms approach have shown some success in reducing addictive behavior [57,58]. Personalized normative feedback (PNF) interventions are a form of PFI premised on the notion that individuals with addictive behavior misjudge the typicality of their attitudes or behaviors relative to their community of peers, and once made aware of this misperception are prone to adjust their attitude or behavior toward those peer norms. The misperception usually involves an individual overestimating the community or peer group norms for some attitude or behavior (e.g. how much alcohol the typical university student consumes) and underestimating how discrepant their own attitudes or behaviors are from those norms (e.g. how much more alcohol they consume than the actual average consumption rate for university students). PNF interventions aimed at correcting such misperceptions (i.e. discrepancies between actual and perceived norms) have shown effectiveness reducing problem drinking and increasing smoking cessation, and several recent studies have extended their application to reducing problem gambling [5962].

Personalized normative feedback was first offered as part of a multicomponent intervention that included in-person motivational interviewing [62]. But recently it has garnered growing interest as a stand-alone intervention delivered, for example, via personal computer (or potentially a mobile device) over the Internet $[63,64]$. Miller and colleagues reviewed 41 studies looking at the effectiveness of PFIs for reducing alcohol misuse, they found that normative feedback - offered in conjunction with other feedback such as didactic/educational information, consideration of practical costs, behavioral strategies - was common to almost all PFI approaches, and that a number of studies had found significant effects for normative feedback as a stand-alone approach [64].

Two recent studies investigated whether PNF could be effective as a stand-alone brief intervention for reducing problem gambling risk. Celio \& Lisman studied the effectiveness of a stand-alone PNF delivered via computer in a laboratory setting with university students [65]. Student participants completed a computerized questionnaire about their gambling behavior and their perceptions of gambling norms among other university students who gamble, and then undertook 2 computer tasks with gambling like features. Students were randomly assigned to a PNF and a non-PNF (attention control) group. The PNF intervention involved students receiving stand-alone computerized feedback on how much they estimated the typical student gambled, how much the typical student actually gambled, and their percentile rank locating their gambling relative to that of other students. The results indicated a positive association between the extent of participants' misperceptions of gambling 
norms and their self-reported gambling - students who reported gambling more were also more likely to overestimate the amount the typical student gambled. Furthermore participants who received PNF showed decreases, 1 week later, in their perception of how much the typical student gambled and in their risk-taking behavior on the two analog measures of gambling. The results point toward the potential "...efficacy of a stand-alone personalized normative feedback intervention as a viable method of eliciting cognitive and behavioral change with regard to gambling..." and the need for further research [65].

Neighbors et al. also found evidence that PNF as a stand-alone intervention was effective for reducing gambling problems and losses and that this reduction was mediated by a reduction in perceived norms of typical student gambling losses [66]. Using PNF "to change normative misperceptions appears to be an effective" intervention for at-risk gambling students and this effect was greater for participants who identified more strongly with their peers, suggesting that gamblers might pay more attention to normative feedback the more particularized it is (e.g. by demographics or by gambling activity type).

The feedback participants received (presented in both text and graphic formats) had four components: (a) the participants' own frequency, expenditure, and time spent gambling; (b) their perceptions of other same-sex students' frequency, expenditure, and time spent gambling; (c) other same-sex students' actual frequency, expenditure, and time spent gambling; and (d) a percentile ranking of the participants' gambling frequency relative to same-sex peers. The authors suggest that, in addition to more specific reference groups, the normative feedback might be even more impactful for at-risk gamblers (who may be prone to overestimating the prevalence among others of the gambling-related negative consequences that they have experienced themselves) if it included normative feedback on problematic behavioral markers, for example indicating that "only $2 \%$ of fellow students had spent more money than intended or gone back to win lost money" [66].

Martens et al. also found evidence of effectiveness for a standalone personalized feedback intervention that provided information on perceived-actual gambling norm discrepancies along with several other types of information (e.g. inventory of recent gambling behaviors, Seven Oaks Gambling Screen categorization, self-reported problems, triggers and myths and cognitions) [67]. Participants were university students at risk for gambling problems. Participants who received personalized feedback reported, at a 3 month follow-up, less money spent gambling and fewer gambling-related problems than the control group. Although the feedback was given in the form of a paper printout, participants read the report themselves as a standalone intervention without any clinician contact.

The studies above took place under laboratory conditions; Auer and Griffiths were given access to real world data to assess the effectiveness of a personalized behavioral feedback system, known as Mentor, available to clientele of one European online gambling site [68]. Users of this website can opt (or not) to sign up to use the mentor program, which, with the push of a button, provides detailed information (numerical, textual, graphical) related to their gambling behavior - wins and losses, game play length, and the number of sessions played in a specified period of time, and trends in their gambling behavior over time (e.g. changes in time spent gambling) including comparison to normative data (other similar type players) - and thus the mentor system extends beyond pop-up messages by providing interactive personalized and detailed user information. The authors compared a group of customers who signed up to use mentor with a similar group (matched on age, gender and gambling intensity) who did not. The group of customers who chose to use the mentor system spent significantly less time and money gambling on the site than the control group who did not use the mentor system. Auer and Griffiths suggest that the mentor system has similar effects seen in systems that ask users to pre-determine time and spending limits, and "facilitates ... setting and adhering to such limits" [68]. While the study focused on users who voluntarily used the mentor system and were not randomly assigned - leaving the possibility that those users may be more responsible in managing their gambling activity to begin with - the results suggest that a system with personalized feedback such as mentor can play a positive role in reducing excessive gambling and encouraging responsible gambling.

Auer et al. provide some additional considerations related to personalized feedback systems [16]. First, they state that gambling limit setting should be a mandatory element in an online gambling site. This is because gambling sites may typically only include voluntary limit setting systems as a result of a legal requirement, and while useful, high-risk and problematic gamblers are not necessarily impacted by voluntary features. Online gambling sites should therefore require users to set time and money-spending limits, although the authors contend that empirical studies into the effectiveness of such mandatory systems is lacking. Secondly, it is important that websites continue to have voluntary limitsetting features in addition to behavioral tracking and personalized feedback systems to help mitigate the risk of problematic gambling. One final recommendation comes from the introduction of optout programs or "exclusions schemes", which may be voluntary or enforced by government regulation [16]. In a voluntary opt-out scheme and individual who has a gambling problem may register for a service (either through a third party or the state) that will prevent the individual from interacting on gambling sites based upon their identity check. Those who know they have a gambling problem may use such a service to prevent their future interactions with gambling sites. The authors point to Denmark as an example, where gambling sites are required to allow users the opportunity to temporarily or permanently exclude themselves from gambling websites. They also note that some countries have legislation enacted (Belgium) or proposed (Netherlands) to permit banning of individuals from online gambling because of gambling addiction. Such legislation is intended to prevent those with uncontrollable gambling problems from harming themselves further. In sum, Auer and colleagues recommend a mix of mandatory and voluntary limit setting in conjunction with personalized feedback and behavioral tracking systems in order to mitigate problem gambling, and suggest that opt-out or exclusionary programs should be available [16].

\section{Treatment?}

The studies above point to the potential efficacy of personalized feedback interventions to moderate gambling involvement and to 
protect low risk and moderate risk gamblers from developing more serious gambling problems. What about those gamblers who are already experiencing more serious gambling problems? Can Internetbased treatment help them? There is growing evidence to suggest that the answer is yes - online therapy can be useful in the treatment of problem gambling both as a stand-alone self-guided intervention and in concert with in-person therapy $[50,69,70]$.

There are several potential advantages to Internet-based interventions including: cost effectiveness (less hiring, training and supervision of clinical staff), convenience (fewer time-related demands and problems related to location/travel, etc.) availability (easily and widely disseminated via technology even to those not actively seeking intervention), flexibility (individually-paced progression through treatment steps and stages of change, modifiable for various levels of need and motivation as well as for different sub-types of gambler), amenability to evaluation (de-identified user data could be employed to evaluate effectiveness of treatment) and privacy and anonymity [50]. Most players do not seek help until they are in serious crisis and even then, less than $10 \%$ of problem gamblers ever seek treatment [71-74]. The prospect of being able to access help relatively easily and anonymously may entice some portion of these individuals to seek self-guided treatment online, and may even encourage some problem gamblers, who wouldn't otherwise, eventually seek faceto-face clinical help [50]. Online treatment modalities may also be particularly effective for adolescents and young adults who are more likely to gamble online than adults and have a preference for accessing services and resources in the online milieu [70].

In addition to personalized feedback interventions, online programs can effectively incorporate other addictions treatment approaches such as motivational interviewing (MI) and cognitive behavioral therapy (CBT). Self-guided programs can help clients determine their level of readiness (based on screening assessments or even actual gambling behavior data) and customize the content accordingly. Increased client participation with tailored online content and exercises may instil greater self-efficacy and commitment to change and are well-suited to helping individuals work through the "stages of change" (pre-contemplation, contemplation, action and maintenance) at their own pace by providing individualized and stage-specific feedback and motivation [75].

Player behavioral tracking information can be incorporated into treatment, either in self-guided interventions or therapist-assisted treatment programs, whether brief or longer term interventions. Behavioral feedback in self-guided programs would resemble the automated algorithm-based modalities discussed in the previous section on RG tools, where personalized feedback would combine self-report screening data (e.g. online diagnostic tests and problem inventories) with objective behavioral tracking feedback (risk level, normative comparisons, pertinent didactic information, etc.) from their actual online gambling to help them make informed decisions and work through appropriately tailored strategies and exercises. Individuals may work through self-help modules on their own or with intermittent therapist contact and support via email or online forum $[50,69]$.

Online behavioral tracking and personalized feedback may also benefit traditional in-person clinical treatment. In-person diagnostic and clinical interviews are bound in part by the limitations of client self-report. Clients may, intentionally or not, misreport their gambling behavior, which can hinder the progress of treatment. Access to objective player behavioral tracking data over time (which in addition to consent of the client would require cooperation of gambling site operators) would enable the clinician to have a more unbiased picture of the client's gambling, including the extent of the problem and potential triggers and patterns [15]. The ability to track the client's gambling behavior over the course of treatment would inform ongoing adjustment of interventions and strategies and could be complemented by stage-appropriate motivational enhancement, PNF (to reduce perceived-actual norm discrepancies) and CBT (to reduce cognitive distortions) techniques.

\section{Conclusion}

On one hand the nature of online gambling appears to invite potential for problematic gambling; on the other hand it is also wellsuited to the incorporation of sophisticated RG tools that can help prevent excessive gambling. There is substantial evidence showing the effectiveness of in-game pop-up messages that disrupt dissociation and encourage player self-appraisal, which when enhanced with behavioral feedback systems can serve to help encourage informed player choice and self-regulation (including limit-setting and selfexclusion) of gambling behavior within safe limits.

The evidence isn't without limitations, as the positive effects of various RG tools are, in practical terms, often rather modest particularly among more intense or problem gamblers, much of the research is colored to some degree by self-selection bias (players who choose to use RG tools may differ in important ways - e.g. motivation, self-awareness - from non-users), and more research is required to discern, for example, which RG tools work best in which combination for which types of games and which types of gamblers. Nevertheless, there is growing recognition of the potential of RG tools that incorporate player behavioral tracking and personalized feedback and that such tools are a win-win for both players and operators as they "can help players enjoy gambling within limits while expanding their lifespan as a player... [which] gives online operators the opportunity to build up a stable and profitable customer relationship" [12].

Although the research is still in early stages, there is also emerging evidence to recommend the effectiveness of online treatment programs for problem gambling (and other addictions) and the potential of incorporating behavioral tracking and personal feedback into such services, whether as part of a self-guided intervention, or as an adjunct to therapist-assisted treatment. Advantages of online treatment include it is cost-effective, amenable to evaluation, as well as flexible and customizable. Additionally, it is convenient to access, widely available, and offers greater privacy and anonymity - features it shares with online gambling and which might appeal to online gamblers, especially tech-savvy youth, who gamble online at higher rates than adults and might feel more comfortable accessing online services. Self-guided intervention programs can help effect positive behavioral change for some (especially those who are reluctant to seek traditional forms of treatment), while others may find greater benefit with therapist-assisted online therapy. Even traditional in-person therapy stands to benefit from access to objective player behavioral data which can help overcome the limits of client self-report by 
Citation: Edgerton JD, Biegun J, Roberts LW. Player Behavioral Tracking and Personalized Feedback in Online Gambling: Implications for Prevention and Treatment of Problem Gambling. J Addiction Prevention. 2016;4(2): 8.

providing clinicians with accurate personalized feedback to inform interventions aimed at changing problematic behaviors, perceptions and cognitions. Certainly, there seems to be much to warrant further research into the potential of player behavioral tracking data and personalized feedback in the treatment of problem gambling.

\section{References}

1. Griffiths M, Wardle H, Orford J, Sproston K, Erens B (2009) Sociodemographic correlates of internet gambling: findings from the 2007 british gambling prevalence survey. Cyberpsychol Behav 12: 199-202.

2. Gainsbury SM, Russell A, Hing N, Wood R, Lubman D, et al. (2015) How the internet is changing gambling: findings from an Australian Prevalence Study. J Gambl Stud 31: 1-15.

3. Griffiths MD, Wood RT (2007) Adolescent internet gambling: preliminary results of a national survey. Educ Health 25: 23-27.

4. Wood RT, Williams RJ (2009) Internet gambling: prevalence, patterns, problems, and policy options. Final report prepared for the Ontario Problem Gambling Research Centre, Guelph, Ontario, Canada.

5. Gambling Participation (2015) 2014-2015 Gambling data \& statistics. Canadian Partnership for Responsible Gambling.

6. Gainsbury S (2012) Internet gambling: current research findings and implications. Springer Briefs in Behavioral Medicine, Springer Science+Business Media, Springer-Verlag, New York, USA

7. Welte JW, Barnes GM, Tidwell MC, Hoffman JH, Wieczorek WF (2015) Gambling and problem gambling in the United States: changes between 1999 and 2013. J Gambl Stud 31: 695-715.

8. Redondo I (2015) Assessing the risks associated with online lottery and casino gambling: a comparative analysis of players' individual characteristics and types of gambling. Int J Ment Health Addict 13: 584-596.

9. Ladouceur R, Blaszczynski A, Lalande DR (2012) Pre-commitment in gambling: a review of the empirical evidence. Int Gambl Stud 12: 215-230.

10. Wood RT, Shorter GW, Griffiths MD (2014) Selecting appropriate gambling features for a specific portfolio of games. Responsible Gambl Rev 1: 51-63.

11. Blaszczynski A, Ladouceur R, Shaffer HJ (2004) A science-based framework for responsible gambling: the Reno model. J Gambl Stud 20: 301-317.

12. Auer M, Griffiths MD (2013) Behavioral tracking tools, regulation, and corporate social responsibility in online gambling. Gaming Law Rev Econ 17 579-583.

13. Blaszczynski A, Sharpe L, Walker M (2001) The assessment of the impact of the reconfiguration on electronic gaming machines as harm minimisation strategies for problem gambling. University of Sydney Gambling Research Unit, Sydney, Australia.

14. Slutske WS, Piasecki TM, Blaszczynski A, Martin NG (2010) Pathologica gambling recovery in the absence of abstinence. Addiction 105: 2169-2175.

15. Gainsbury SM (2011) Player account-based gambling: potentials for behaviour-based research methodologies. Int Gambl Stud 11: 153-171.

16. Auer M, Littler A, Griffiths MD (2015) Legal aspects of responsible gaming pre-commitment and personal feedback initiatives. Gaming Law Rev Econ 19: 444-456.

17. Richard DC, Blaszczynski A, Nower L (2014) The Wiley-Blackwell handbook of disordered gambling. West Sussex, Wiley-Blackwell, UK

18. Zangeneh M, Blaszczynski A, Turner N (2008) In the pursuit of winning problem gambling theory, research and treatment, Springer, New York, USA

19. Gainsbury S (2014) Internet gaming and disordered gambling. In: Richard DC, Blaszczynski A, Nower L (Eds), The Wiley-Blackwell handbook of disordered gambling. West Sussex, Wiley-Blackwell, UK.

20. Wood R, Williams R, Parke J (2012) The relationship between internet gambling and problem gambling. In: Williams RJ, Wood RT, Parke J (Eds), Routledge international handbook of internet gambling. Taylor \& Francis Ltd, Routledge, London, UK, pp. 200-211.

21. Gainsbury SM (2015) Online gambling addiction: the relationship between internet gambling and disordered Gambling. Curr Addict Rep 2: 185-193.

22. Griffiths M (2003) Internet gambling: issues, concerns and recommendations. Cyberpsychol Behav 6: 557-568.

23. Wood RT, Williams RJ (2007) Problem gambling on the internet: implications for Internet gambling policy in North America. New Media Soc 9: 520-542.

24. Monaghan S (2009) Responsible gambling strategies for internet gambling the theoretical and empirical base of using pop-up messages to encourage self-awareness. Comput Human Behav 25: 202-207.

25. Welte JW, Barnes GM, Tidwell MC, Hoffman JH (2009) The association of form of gambling with problem gambling among American youth. Psychol Addict Behav 23: 105-112.

26. Haefeli J, Lischer S, Schwarz J (2011) Early detection items and responsible gambling features for online gambling. Int Gambl Stud 11: 273-288.

27. Harris A, Griffiths MD (2016) A critical review of the harm-minimisation tools available for electronic gambling. J Gambl Stud [Epub ahead of print].

28. Philander KS, MacKay TL (2014) Online gambling participation and problem gambling severity: is there a causal relationship? Int Gambl Stud 14: 217-227.

29. Diskin KM, Hodgins DC (2001) Narrowed focus and dissociative experiences in a community sample of experienced video lottery gamblers. Can J Behav Sci 33: 58-64.

30. Grant JE, Kim SW (2003) Dissociative symptoms in pathological gambling Psychopathology 36: 200-203.

31. Monaghan S, Blaszczynski A (2010) Impact of mode of display and message content of responsible gambling signs for electronic gaming machines on regular gamblers. J Gambl Stud 26: 67-88.

32. Floyd K, Whelan JP, Meyers AW (2006) Use of warning messages to modify gambling beliefs and behavior in a laboratory investigation. Psychol Addict Behav 20: 69-74.

33. Ladouceur R, Sevigny S (2003) Interactive messages on video lottery terminals and persistence in gambling. Gambl Res: J Natl Assoc Gambl Stud 15: $45-50$.

34. Cloutier M, Ladouceur R, Sévigny S (2006) Responsible gambling tools: popup messages and pauses on video lottery terminals. J Psychol 140: 434-438.

35. Wood RT, Griffiths MD (2010) Social responsibility in online gambling: voluntary limit setting. World Online Gambl Law Rep 9: 10-11.

36. Nower L, Blaszczynski A (2010) Gambling motivations, money-limiting strategies, and precommitment preferences of problem versus non-problem gamblers. J Gambl Stud 26: 361-372.

37. Broda A, LaPlante DA, Nelson SE, LaBrie RA, Bosworth LB, et al. (2008) Virtual harm reduction efforts for internet gambling: effects of deposit limits on actual internet sports gambling behavior. Harm Reduct J 5: 27-36

38. Blaszczynski A, Gainsbury S, Karlov L (2014) Blue gum gaming machine: an evaluation of responsible gambling features. J Gambl Stud 30: 697-712.

39. Kim HS, Wohl MJ, Stewart MJ, Sztainert T, Gainsbury SM (2014) Limit your time, gamble responsibly: setting a time limit (via pop-up message) on an electronic gaming machine reduces time on device. Int Gambl Stud 14: 266 278.

40. Stewart MJ, Wohl MJ (2013) Pop-up messages, dissociation, and craving how monetary limit reminders facilitate adherence in a session of slot machine gambling. Psychol Addict Behav 27: 268-273.

41. Wohl MJ, Gainsbury S, Stewart MJ, Sztainert T (2013) Facilitating responsible gambling: the relative effectiveness of education-based animation and monetary limit setting pop-up messages among electronic gaming machine players. J Gambl Stud 29: 703-717. 
Citation: Edgerton JD, Biegun J, Roberts LW. Player Behavioral Tracking and Personalized Feedback in Online Gambling: Implications for Prevention and Treatment of Problem Gambling. J Addiction Prevention. 2016;4(2): 8.

42. Auer M, Malischnig D, Griffiths M (2014) Is "pop-up" messaging in online slot machine gambling effective as a responsible gambling strategy? J Gamb Issues 29: 1-10.

43. Auer MM, Griffiths MD (2015) Testing normative and self-appraisal feedback in an online slot-machine pop-up in a real-world setting. Front Psychol 6: 339.

44. Adami N, Benini S, Boschetti A, Canini L, Maione F, et al. (2013) Markers of unsustainable gambling for early detection of at-risk online gamblers. In Gambl Stud 13: 188-204

45. Boldero JM, Bell RC, Moore SM (2010) Do gambling activity patterns predict gambling problems? A latent class analysis of gambling forms among Australian youth. Int Gambl Stud 10: 151-163.

46. Braverman J, Shaffer HJ (2012) How do gamblers start gambling: identifying behavioural markers for high-risk internet gambling. Eur J Public Health 22 273-278.

47. Delfabbro P, King DL, Griffiths M (2012) Behavioural profiling of problem gamblers: a summary and review. Int Gambl Stud 12: 349-366.

48. LaBrie R, Shaffer HJ (2011) Identifying behavioral markers of disordered internet sports gambling. Addict Res Theory 19: 56-65

49. Schrans T, Schellinck T, Walsh G, Focal Research Consultants Ltd (2000) Nova Scotia regular video lottery players follow-up study: a comparative analysis of problem development and resolution. Nova Scotia Department of Health, Addictions Services.

50. Gainsbury S, Blaszczynski A (2011) Online self-guided interventions for the treatment of problem gambling. Int Gambl Stud 11: 289-308.

51. Parke J, Rigbye J, Parke A, Sjenitzer J, Wood R, et al. (2007) An exploratory investigation into attitudes and behaviours of internet casino and poke players. Commissioned by eCOGRA (e-Commerce and Online Gaming Regulation and Assurance)

52. Bernhard BJ (2007) The voices of vices: Sociological perspectives on the pathological gambling entry in the diagnostic and statistical manual of mental disorders. Am Behav Sci 51: 8-32.

53. Blaszczynski A, Ladouceur R, Nower L, Shaffer H (2005) Informed choice and gambling: principles for consumer protection. Current issues, Australian Gaming Council, Australia.

54. Reith G (2008) Reflections on responsibility. J Gambl Issues 22: 149-155.

55. Wohl MJ, Parush A, Kim HS, Warren K (2014) Building it better: applying human-computer interaction and persuasive system design principles to a monetary limit tool improves responsible gambling. Comput Human Behav 37: $124-132$.

56. Wood RT, Wohl MJ (2015) Assessing the effectiveness of a responsible gambling behavioural feedback tool for reducing the gambling expenditure of at risk-players. Int Gambl Stud 15: 1-16.

57. Bandura A (1971) Social learning theory. General Learning Press, New York, USA.

58. Berkowitz AD (2005) An overview of the social norms approach. In: Lederman LC, Stewart LP (Eds), Changing the culture of college drinking: a socially situated health communication campaign. Hampton Press Inc, New York USA, pp. 193-222.
59. Labrie JW, Lewis MA, Atkins DC, Neighbors C, Zheng C, et al. (2013) RCT of web-based personalized normative feedback for college drinking prevention: are typical student norms good enough? J Consult Clin Psychol 81: 10741086.

60. Bryant ZE, Henslee AM, Correia CJ (2013) Testing the effects of e-mailed personalized feedback on risky alcohol use among college students. Addict Behav 38: 2563-2567.

61. van den Putte B, Yzer M, Willemsen MC, de Bruijn GJ (2009) The effects of smoking self-identity and quitting self-identity on attempts to quit smoking. Health Psychol 28: 535-544.

62. Marchica L, Derevensky JL (2016) Examining personalized feedback interventions for gambling disorders: a systematic review. J Behav Addict 5: $1-10$.

63. Collins SE, Kirouac M, Lewis MA, Witkiewitz K, Carey KB (2014) Randomized controlled trial of web-based decisional balance feedback and personalized normative feedback for college drinkers. J Stud Alcohol Drugs 75: 982-992.

64. Miller MB, Leffingwell T, Claborn K, Meier E, Walters S, et al. (2013) Personalized feedback interventions for college alcohol misuse: an update of Walters \& Neighbors (2005). Psychol Addict Behav 27: 909-920.

65. Celio MA, Lisman SA (2014) Examining the efficacy of a personalized normative feedback intervention to reduce college student gambling. J Am Coll Health 62: 154-164.

66. Neighbors C, Rodriguez LM, Rinker DV, Gonzales RG, Agana M, et al. (2015) Efficacy of personalized normative feedback as a brief intervention for college student gambling: a randomized controlled trial. J Consult Clin Psychol 83: 500-511.

67. Martens MP, Arterberry BJ, Takamatsu SK, Masters J, Dude K (2015) The efficacy of a personalized feedback-only intervention for at-risk college gamblers. J Consult Clin Psychol 83: 494-499.

68. Auer MM, Griffiths MD (2015) The use of personalized behavioral feedback for online gamblers: an empirical study. Front Psychol 6: 1406.

69. Gainsbury S, Blaszczynski A (2011) A systematic review of internet-based therapy for the treatment of addictions. Clin Psychol Rev 31: 490-498.

70. Monaghan S, Wood RT (2010) Internet-based interventions for youth dealing with gambling problems. Int J Adolesc Med Health 22: 113-128.

71. Clarke D, Abbott M, DeSouza R, Bellringer M (2007) An overview of help seeking by problem gamblers and their families including barriers to and relevance of services. Int J Ment Health Addict 5: 292-306.

72. Suurvali H, Hodgins DC, Cunningham JA (2010) Motivators for resolving or seeking help for gambling problems: a review of the empirical literature. $J$ Gambl Stud 26: 1-33.

73. Cunningham JA (2005) Little use of treatment among problem gamblers. Psychiatr Serv 56: 1024-1025

74. Slutske WS, Blaszczynski A, Martin NG (2009) Sex differences in the rates of recovery, treatment-seeking, and natural recovery in pathological gambling: results from an Australian community-based twin survey. Twin Res Hum Genet 12: 425-432.

75. Prochaska JO, Prochaska JM (1999) Why don't continents move? Why don't people change? J Psychother Integr 9: 83-102. 